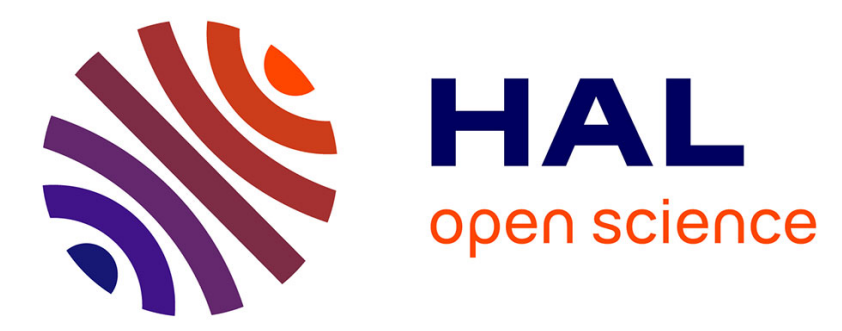

\title{
PISTIL interferometry: a modular, plug-and-play metrology mean for diagnosis of coherently combined laser arrays
}

Bastien Rouzé, Jérôme Primot, Frédéric Zamkotsian, Patrick Lanzoni, Cindy Bellanger

\section{To cite this version:}

Bastien Rouzé, Jérôme Primot, Frédéric Zamkotsian, Patrick Lanzoni, Cindy Bellanger. PISTIL interferometry: a modular, plug-and-play metrology mean for diagnosis of coherently combined laser arrays. SPIE Photonics West 2021 - SPIE LASE, Mar 2021, Online, United States. pp.116651K, 10.1117/12.2582509. hal-03167720

\section{HAL Id: hal-03167720 \\ https://hal.science/hal-03167720}

Submitted on 12 Mar 2021

HAL is a multi-disciplinary open access archive for the deposit and dissemination of scientific research documents, whether they are published or not. The documents may come from teaching and research institutions in France or abroad, or from public or private research centers.
L'archive ouverte pluridisciplinaire HAL, est destinée au dépôt et à la diffusion de documents scientifiques de niveau recherche, publiés ou non, émanant des établissements d'enseignement et de recherche français ou étrangers, des laboratoires publics ou privés. 


\title{
PISTIL interferometry: a modular, plug-and-play metrology mean for diagnosis of coherently combined laser arrays
}

\author{
Bastien Rouzé $^{1 \mathrm{a}}$, Jérôme Primot ${ }^{\mathrm{a}}$, Frédéric Zamkotsian ${ }^{\mathrm{b}}$, Patrick Lanzoni ${ }^{\mathrm{b}}$, Cindy Bellanger $^{\mathrm{a}}$ \\ ${ }^{a}$ DOTA, ONERA, Université Paris-Saclay, BP 80100, 91123 Palaiseau cedex, France \\ b Aix Marseille Univ, CNRS, CNES, LAM - Laboratoire d'Astrophysique de Marseille, 38 Rue \\ Frédéric Joliot Curie, 13388 Marseille Cedex 13, France
}

\begin{abstract}
PISTIL (PISton and TILt) interferometry is a segmented wavefront metrology technique that can fulfill the role of being an independent phase analyzer for tiled laser arrays used in coherent beam combining (CBC). It presents a plug-and-play characteristics enabling others research or industrial applications such as metrology of segmented mirrors, MOEMS or measurement standards. It can operate onto complex optical benches. Alongside the PISTIL concept, we developed methods for phase extraction and meta-analysis, with best accuracy to rightfully address an end user needs in term of segmented wavefront diagnosis. We demonstrate those functionalities onto the HIBISCUS optical testbed equipped with a segmented mirror, specifically designed test data analysis pipelines and improve the control-command based on PISTIL wavefront analysis. In the current configuration, it can emulate CBC near field piston and tilt variations.
\end{abstract}

Keywords: PISTIL, PISTIL interferometry, segmented wavefront, wavefront analyzer, coherent beam combining

\section{INTRODUCTION}

The research for ultra-intense laser sources is now achieving tremendous performance worldwide and generates innovative ideas [1, 2]. The coherent beam combining (CBC) [2-4], of several elementary laser sources is one of the key architecture for the design of these new high-power, high-radiance laser sources. These elementary sources are often fiber amplifiers derived from a MOPA (Master Oscillator Power Amplifier) working in nominal regime. In spatial CBC systems, the laser pupil is tiled in several collimated sub beams that generate in near field a segmented wavefront made of the phases of all those sub beams. Speckle is seen if all those phases are randomly set (Figure 1-a). CBC is achieved when this meshed laser pupil is phased, propagates and ends up interfering constructively (Figure 1-b), which maximizes the output power on a unique, stable, spatial position.

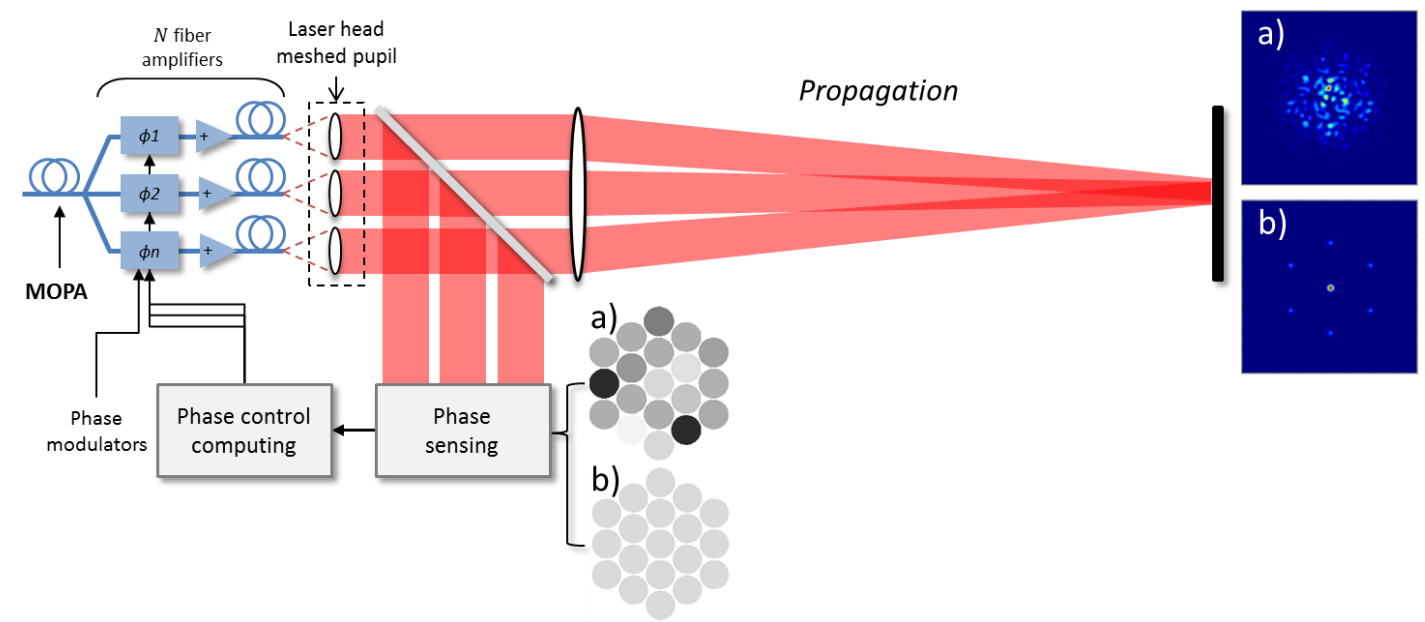

Figure 1. Layout of a CBC system with a tiled aperture (hexagonal mesh). (a) Speckle is seen in far field when phases are neither analyzed nor controlled. (b) Constructive interference in Far field obtained via a phase-locked flat wavefront.

${ }^{1}$ bastien.rouze@onera.fr 
The sub beams phases thus need to be phase-locked altogether in a specific scheme, often to get a flat wavefront to maximize output power [4, 5], to get vortex wavefronts to generate Bessel beams [6-8], or other beam shapes [9]. The use of the laser depends on the research or industrial needs.

If several phase control schemes exist as of today, such as SPGD, LOCSET, interferometry-based or phase mask means [10-15], the large increase in combined sources [16-18], overall optical bench management, phase perturbations (turbulence, blooming) [19], made it necessary to develop an external independent phase control mean.

Probing very precisely the phase of each of the sub beams will allow to optimize the parameters of the phase control loop and to refine the alignment of the optical head. It will also allow to evaluate the recombination obtained, and thus to estimate the quality of the beam produced. Thus, phase piston and tip/tilt (fiber inclination) of each fiber in near field must be estimated by a non-invasive, self-referenced mean with high trueness and precision. In addition, modularity and compactness would be a plus to accommodate with various optical configurations, and/or complex environment (IR, partitioned benches or clean rooms).

The PISton and TILt (PISTIL) interferometry [20-23] has been developed, specifically designed for precise measurement of phase delays and inclination of any type of regular segmented wavefront, as in CBC. Its plug-and-play characteristics in operation make it employable for a wide variety of procedures beside CBC, such as segmented mirror or segmented phase screen metrology, overall on complex bench work, whereas reference flat based means cannot access.

In this paper, the main principle of the PISTIL interferometry is firstly presented in Section 2. We discuss about its hardware and software modularity. Then, we present in Section 3 alongside the PISTIL meta-analysis toolbox, a workbench named HIBISCUS. This bench is not only a modular laboratory for PISTIL hardware and software research and development, but also a mean of testing phase control systems whose specs and sub-specs variations are and will be given by PISTIL analysis feedbacks without having to overuse the possibly complex laser system.

\section{PISTIL INTERFEROMETRY}

\subsection{Principle}

The PISTIL interferometry is a member of the multilateral shearing interferometry family. It is specifically designed for measuring pistons $P$ and tips/tilts $t_{X, Y}$ of various elements of a meshed segmented wavefront, such as Cartesian or hexagonal meshes used in the CBC domain. PISTIL is designed to sense the neighbor difference in pistons $\Delta P$ and tips/tilts $\Delta t_{X, Y}$ of the tiled pupil made of elementary sources or sub beams (Section 2.1), and then reconstitute the segmented wavefront (Section 2.2).

To simplify the description, the process is described here with only two elements from a hexagonal mesh (Figure 2, left). The main steps are the following:

- Lacunarization of the segmented surface with a hole mask to select the center part of the beam for each segment and create independent sub-wavefronts. The aim is to have a sub-wavefront diameter lower than half the distance between two adjacent sub-wavefronts,

- The lacunarized wavefront goes through a diffracting component, such as a grating for example. The diffracting component (with period $p_{g}$ ) produces lateral sheared replicas of the incident wavefront,

- A camera is finally placed at a distance allowing a perfect overlap between replicas of adjacent sub beams, allowed thanks to the lacunarzation. If necessary, reimaging the pistilogram is possible with an afocal system.

Each fringe pattern resulting from 2-wave interferences (Figure 2, orange circles) gives clear information on the relative piston and tip/tilt (the three main phase defaults in CBC or segmented wavefront analysis) between two adjacent elements in a simple manner (Figure 2, right):

- $\quad$ Piston difference $\Delta P=P_{1}-P_{2}$ translates into a fringe pattern shift,

- Tip difference $\Delta t_{X}=t_{X 1}-t_{X 2}$ (along a $x$ direction) translates into a fringe pattern frequency change,

- Tilt difference $\Delta t_{Y}=t_{Y 1}-t_{Y 2}$ (along a $y$ direction, orthogonal to $x$ ) translates into a fringe pattern orientation change. 
The following mathematical formula describes the fringes pattern observed for a wavelength $\lambda$ :

$$
I(x, y) \propto 1+\cos \left(2 \pi\left(\frac{2 x}{p_{g}}+\frac{\Delta t_{X} x}{\lambda}+\frac{\Delta t_{Y} y}{\lambda}+\frac{\Delta P}{\lambda}\right)\right)
$$

Within Figure 3 is shown the typical complete interferogram, called "pistilogram" obtained by simulation, on 37 hexagonal flat beams. In this configuration, there are three types of two-wave interference fringe patterns, along the three main hexagon axes $\left(0^{\circ},-120^{\circ}\right.$ and $\left.+120^{\circ}\right)$. In such situation, 90 fringe patterns, called "petals" are available for measurement. In a general way, for the hexagonal shape of $R$ rings ( 2 or 3 in this paper) containing $3 R^{2}+3 R+1$ sub beams, a total number of $9 R^{2}+3 R$ fringes can be measured.

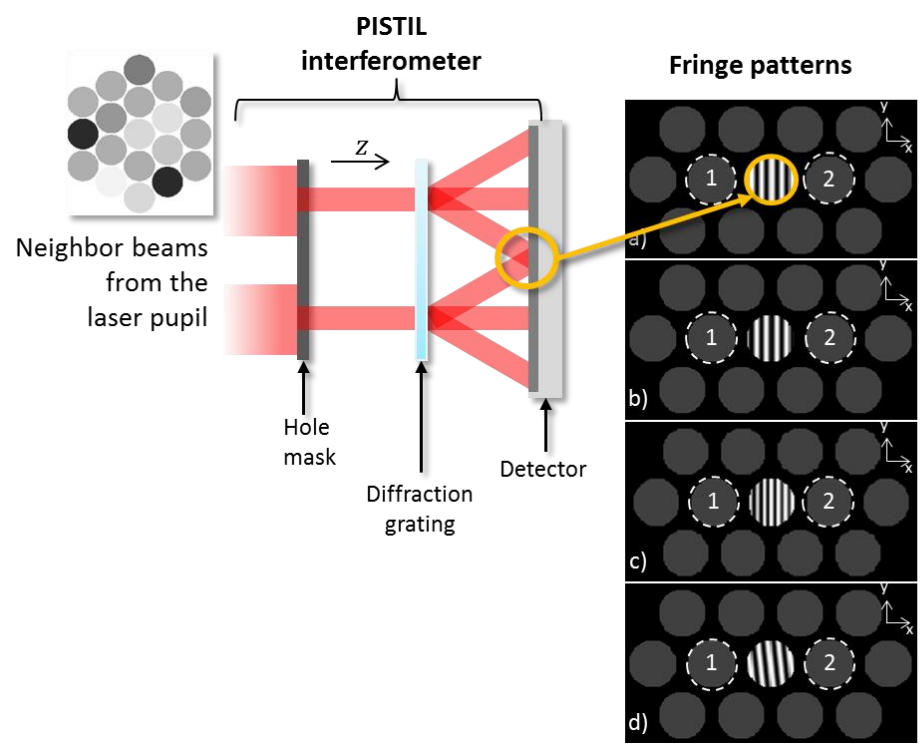

\section{Phase/relative phase defaults}
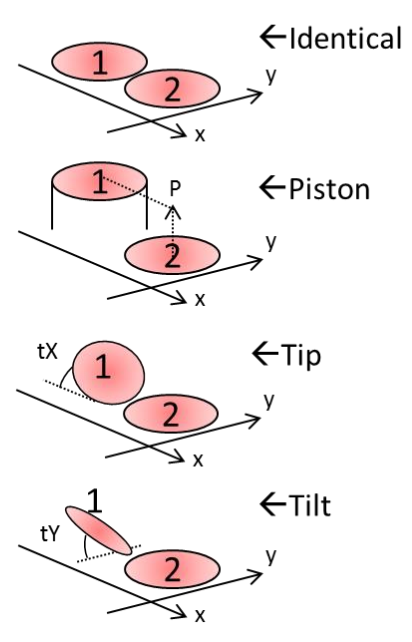

Figure 2. Concept of the PISTIL interferometry technique applied to two neighboring beams sampled from the laser pupil for a tutorial example.

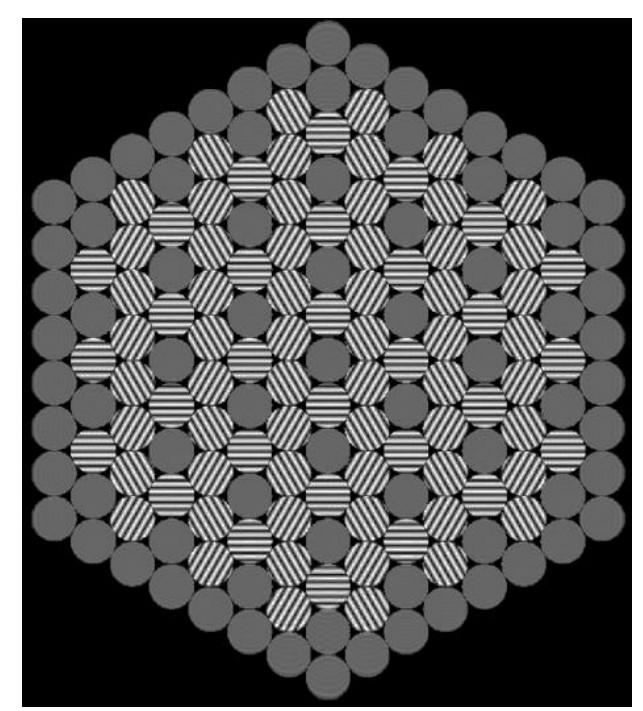

Figure 3. Theoretical pistilogram for a hexagonal mesh pupil of 37 elements, having 90 fringe patterns to recover phase differences from. 


\subsection{Segmented wavefront reconstruction}

The pistilogram recorded contains several fringes pattern presenting different orientations, encircled in Figure 4-a. Those patterns contain, relatively to a reference pattern, information about piston, tip or tilt differences. They can be processed by calculating the Fourier transform of the pistilogram, a literature method in interferometry [24]. The spatial spectrum is mainly made up of three pairs of harmonics relative to the three natural hexagon directions (Figure 4-b).

A parallel processing is then carried out on each of the harmonics of interest: the area corresponding to each harmonic is selected and a reverse Fourier transform is performed to estimate the phase gradient in all three directions. The resulting gradients are then combined to form a phase gradient map, with sub-areas corresponding to the phase difference between each segment and its neighbor (Figure 5, left). Then, relative piston, tip and tilt are extracted by projection with reference functions for each "phase petal".
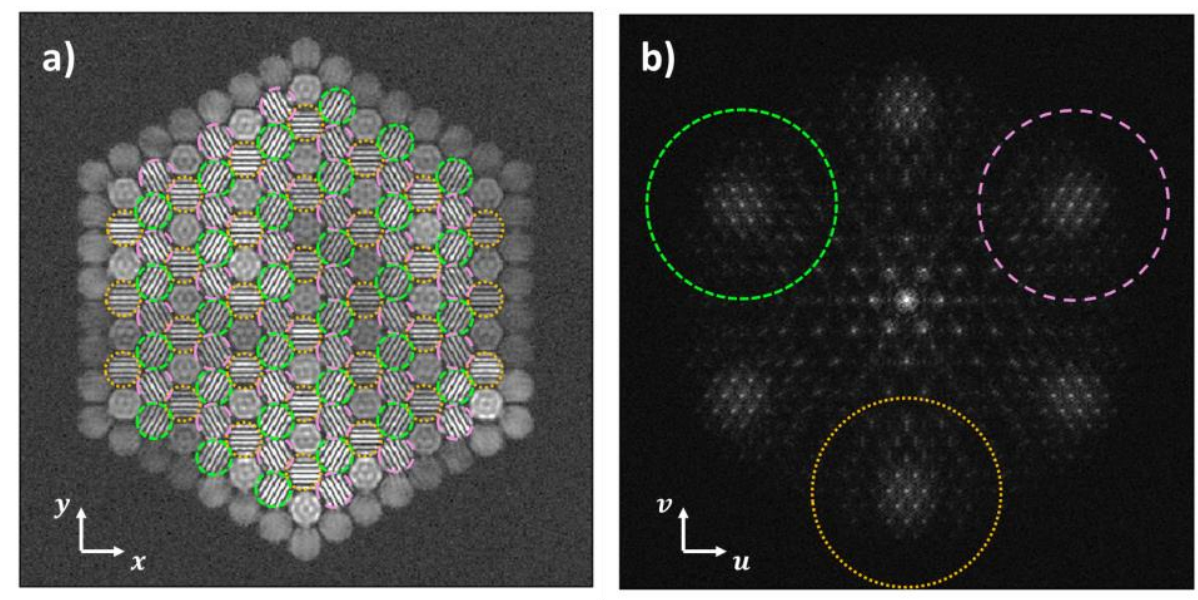

Figure 4. (a) Simulation of a real pistilogram obtained from a laser array of 37 elements. (b) Fourier Transform of the pistilogram, with related fringe Fourier information encircled in both representations.

Finally, the absolute information of piston and tip/tilt for each sub beam is retrieved by integration of these phase petals, using a general inverse matrix method [25]. The large amount of redundant information is then used to reinforce the quality of the reconstruction. An example of such reconstruction is shown in Figure 5 (right), for the same number of fibers (37) as previously, and various input of pistons for each segment, which is the common aberration to be sensed and corrected for.
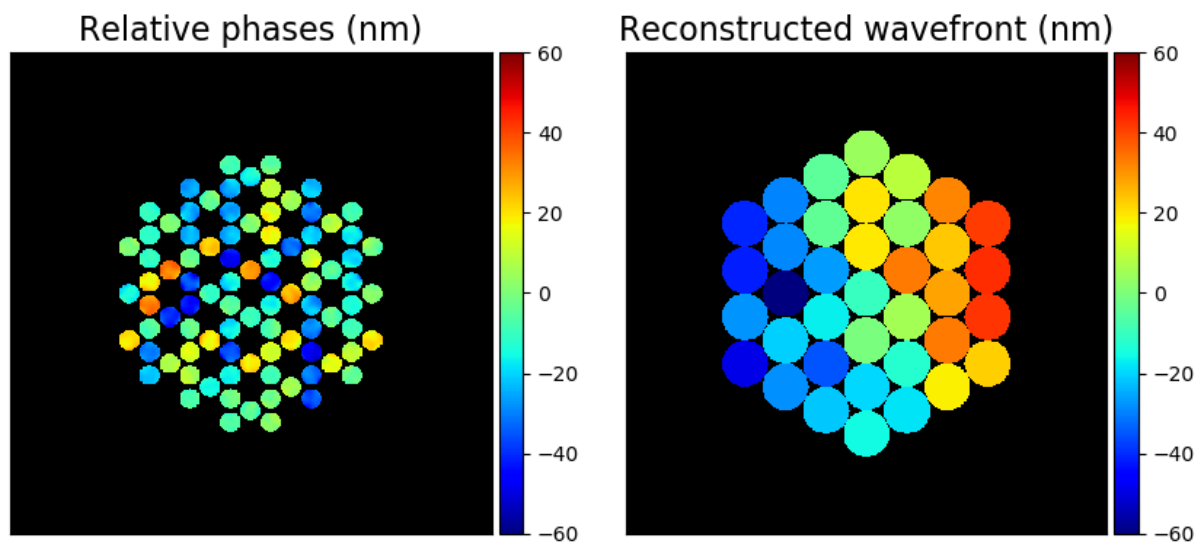

Figure 5. Left panel: Phase petals extracted from the fringes by the Fourier transform algorithm. Right panel: piston segmented wavefront reconstructed by application of the general inversion matrix method.

This process has been successfully tested in [21] and [22, 23] for a two-wavelength interferometry variation (only requiring to record two color pistilograms), on segmented deformable mirrors (DM). Speaking of which, this technology is also naturally in the industrial scope of the PISTIL interferometry. 


\subsection{Side properties of the PISTIL interferometry}

For a considered application, depending on the characteristics of the considered aberrations (amplitude, cadence, interferometer manufacture...), some preliminary calibration steps can be performed in the metrology laboratory. It consists in taking a measurement with a reference wavefront. This can be either a perfect plane produced by a highprecision collimator or a specific wavefront, in accordance with the ideal wavefront shape required for the experiment. It should be noted that this step of calibration in the laboratory is necessary and sufficient for piston measurement. If only the quality of a phase-locked scheme is required by the CBC laser end users, only a mean pistilogram taken from the closed loop measurement will be required, without the use of any additional reference arm, whose role is now assumed by the self-referenced PISTIL.

Depending on the situation, it is necessary to match the pistilogram dimension to the focal plane array, because of the specification or to take benefit of sensor fps boost by limiting the region of interest of the sensor. So, the geometrical pistilogram plane is reimaged into a pupil plane with an afocal system. It switches the location of the optimal distance toward in between the hole mask and the diffraction grating.

Moreover, there may be a magnification between the laser pupil and the PISTIL mask, and a magnification of the optical elements placed between the mask and the detector. In such scenarios, Gouy's theorem insures the conservation of optical path difference during propagation, thus the piston calibration is insensitive to pure magnification. This makes PISTIL very adaptive on various optical benches. Whether piston is not affected, tip/tilts will be (Figure 6). Then, if the measurement of tips/tilts is also of interest, an additional calibration step is required in the laboratory, for example with a tilt controlled plane wave.
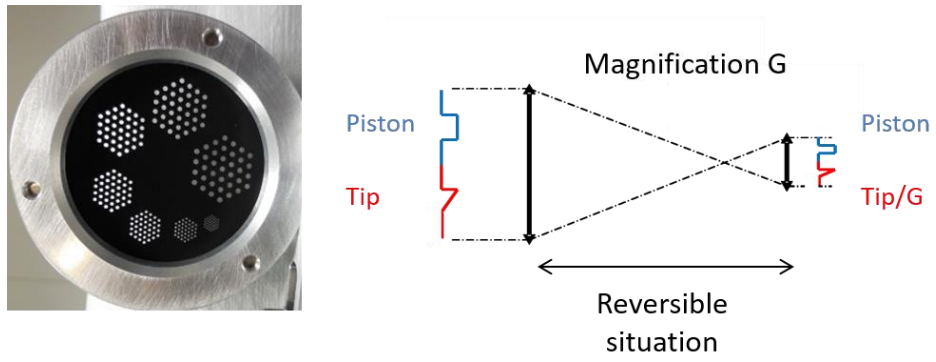

Figure 6. The scheme represents the effect of an afocal system with magnification $\mathrm{G}$ on piston (blue) and tip (red) aberrations. The piston optical path remains unchanged while the tip is weighted by G. On the left, an experimental PISTIL hole mask that can match different magnifications, and can be easily aligned.

About the meshes, the PISTIL interferometer essentially depends on the pattern of the segmented wavefront and the one of its diffraction grating. Therefore, it is possible to calibrate on fairly regular segmented surfaces by designing the appropriate diffraction grating for the desired application. For a hexagonal mesh (as presented in Figure 2), a 2D diffraction grating is required that generates replicas towards the 6 neighbors of the considered element. For a square mesh, it will only be necessary to generate 4 replicas (see Figure 7). Missing tiles are not of an issue.
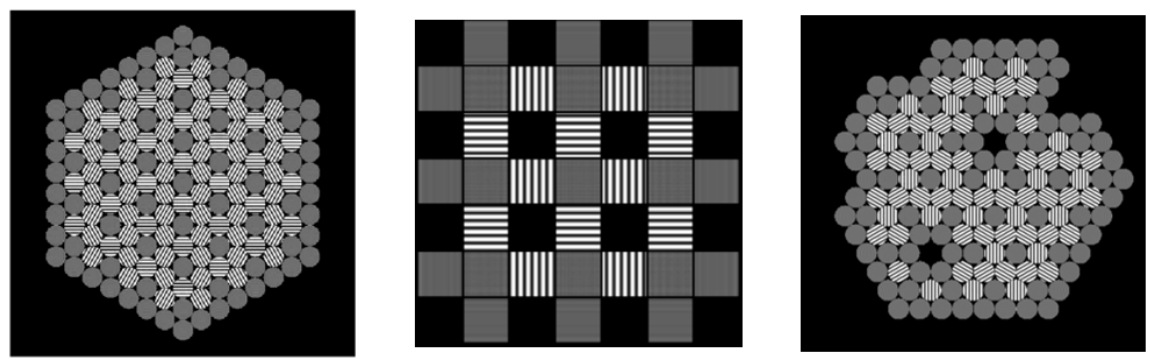

Figure 7. The PISTIL can be applied to several tiled apertures, such as hexagonal or cartesian meshes. Also, it can be applied if there is missing tiles in the mesh. 


\section{HIBISCUS: A LABORATORY FOR WAVEFRONT ANALYSIS, WAVEFRONT CONTROL AND WAVEFRONT SENSOR PROTOTYPE DESIGN}

We developed a demonstrative bench into a segmented wavefront sensing laboratory called HIBISCUS (see in Figure 8). This modular worktop offers several operating modes:

- A research mode, where we can test new hardware or software setups for improving or extending the PISTIL interferometry capabilities.

- A learning mode, where a PISTIL interferometer is deployed onto a real CBC laser chain, and carries out in situ measurements and extractions afterwards. These data are shared with the end user and packaged back to the HIBISCUS library.

- An open/closed loop stimuli mode: a wavefront variation scenario can be emulated on the workbench thanks to a segmented deformable mirror IRIS-AO PTT-111, with the corresponding parameters to the one of the laser diagnosed. A PISTIL interferometer can be plugged in to probe the correction made to the scenario. Additional arms can be added to implement other analyzers.

The IRIS-AO PTT-111 deformable mirror (DM) is constituted of 37 hexagonal segments that can move in piston (max stroke $1.5 \mu \mathrm{m})$. It is a perfect emulator candidate of a CBC laser near field. This DM has been extensively characterized in previous works $[23,26]$.

The HIBISCUS bench is a well-adapted metrology bench which serves for practical manufacture of PISTIL interferometers and design of wavefront control schemes for active/adaptive optics or CBC phase feedback loops. In addition, it may go beyond this CBC representative laboratory framework. Transverse applications can be thought of, such as MOEMS metrology, commercial and experimental segmented mirrors (such the one developed for wavefront correction in astronomy), other blazed gratings-like optical surfaces, or in the industry. The operating scheme would be the same: a PISTIL will probe the element and report additional knowledge to the end user and to the HIBISCUS laboratory. The bench could be modified to accommodate the segmented surface to be tested instead of the current DM.

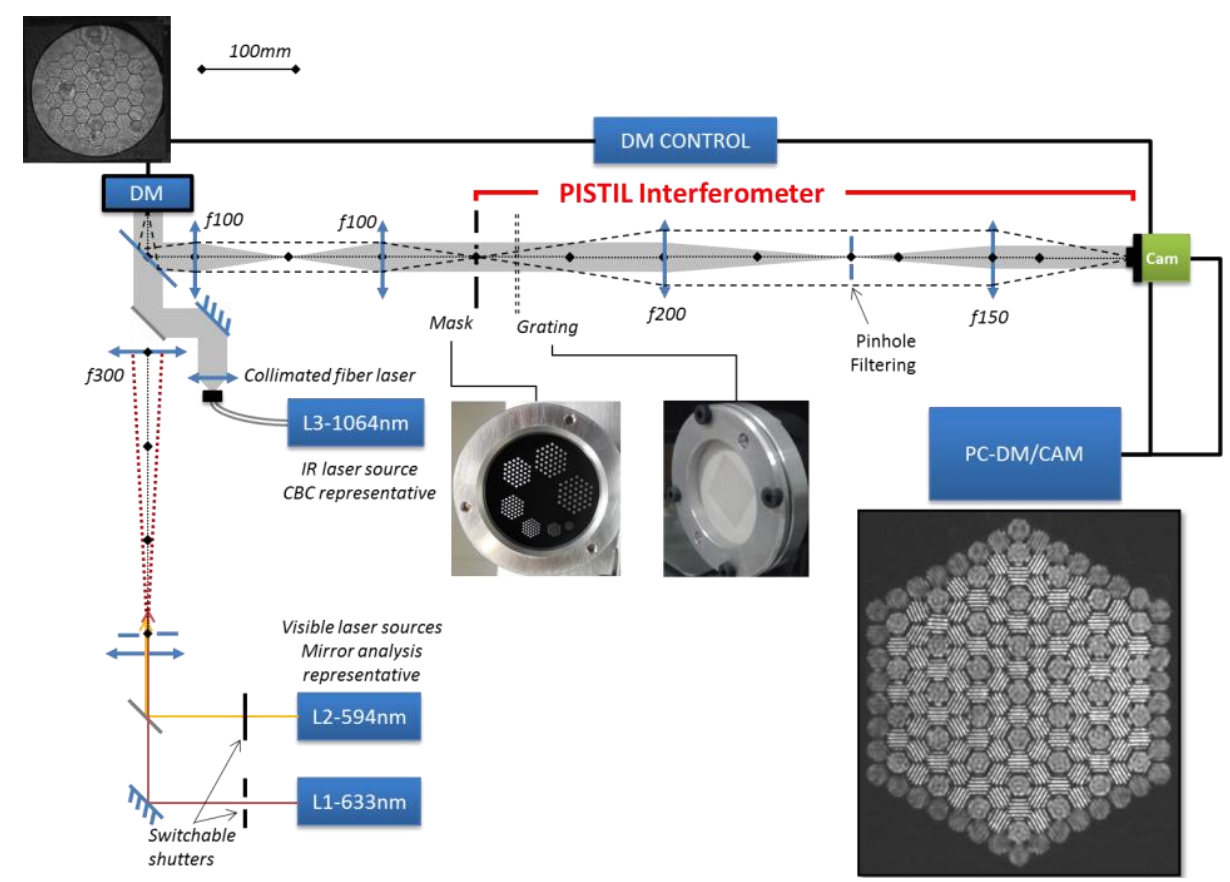

Figure 8. HIBISCUS bench for PISTIL interferometry research tests and manufacture. True pistilogram shows clear resemblance to theory. 
Three laser sources, two HeNe lasers and a collimated diode $\left(\lambda_{1}=633 \mathrm{~nm}, \lambda_{2}=594 \mathrm{~nm}, \lambda_{3}=1064 \mathrm{~nm}\right)$, are installed on the bench. The two HeNe lasers serve to perform Two-Wavelength PISTIL interferometry for laboratory metrology purposes, while the IR laser allows us to test all our optical components in NIR CBC-like conditions. The DM segmented wavefront is conjugated by two afocal systems to test magnification changes at the input of the system, and in reimaging optics.

The standard PISTIL hole mask has a pitch of $D=606 \mu \mathrm{m}$ with a hole diameter of $300 \mu \mathrm{m}$, several magnifications available (Figure 6 and Figure 7). The diffractive element is an intensity diffraction grating (or a phase diffraction grating with $\left.\lambda_{3}\right)$ having a period of $p_{g}=97 \mu \mathrm{m}$. The camera is a JAI SP-5000-CXP4 $(2580 \times 2048$ pixels, pitch of $5 \mu \mathrm{m}$, standard framerate up to $250 \mathrm{~Hz}$, boosted up to $1600 \mathrm{~Hz}$ in $320 \times 320$ pixels windowed mode). In practice, all these elements are mounted onto an optical rail (Figure 9), which facilitates optical alignment, maintenance, and equipment changes. Hole mask and grating can be manufactured industrially, and materials can be chosen according to the application, therefore focusing on their impact onto the pistilogram image quality.

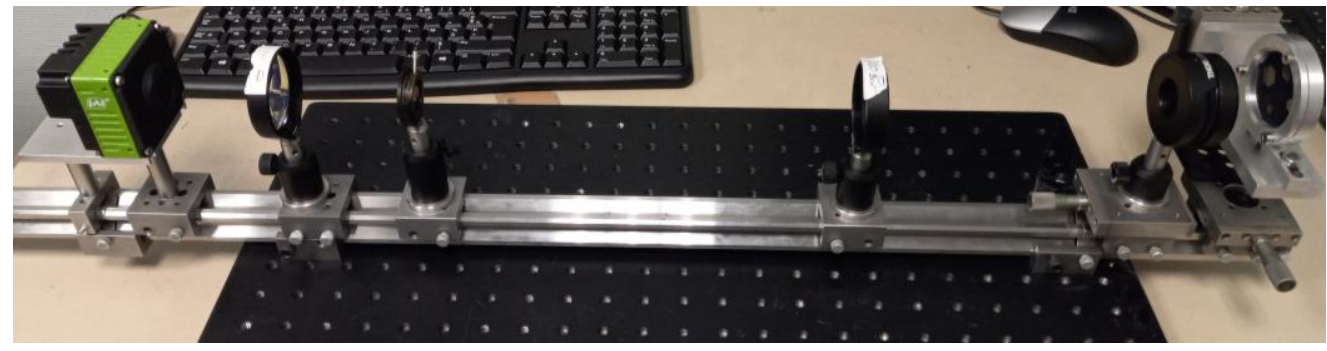

Figure 9. Transportable prototype of the PISTIL interferometer. The rail is dismounted from its support and placed on the end user optical bench.

\section{PISTIL INTERFEROMETRY DIAGNOSIS TOOLBOXES ON A CBC NEAR FIELD EMULATED BY A SEGMENTED MIRROR}

We emulated phase temporal evolution of a laser in a very low rms closed loop by tracking the mirror stability set electronically flat and at rest. Pistilogram sequences are recorded and then computed by the pipeline described in Section 2, to derive temporal evolution of the segmented wavefront. We thus are sensitive to all external noise sources that impact the stability test: $50 \mathrm{~Hz}$ peak (electrical network), thermo-mechanical disturbances (camera heating), acoustic noises (computer fans). This result in small global tip/tilt shifts of the sensed wavefront, as displayed in Figure 10 and Figure 11, which also presents piston per segment tracking diagnosis. The average piston RMS value is $\lambda_{3} / 200$, and the worst value of $\lambda_{3} / 150$ for external segments. It is only due to the erratic tip-tilt caused by the laboratory noise sources. It gives the PISTIL interferometer sensed piston noise floor in dynamic experimental conditions. If one removes the tip-tilt aberrations, RMS value drops down to $\lambda_{3} / 880$ ( $1 \mathrm{~nm}$ floor), therefore leading to noticeably accurate static analysis.

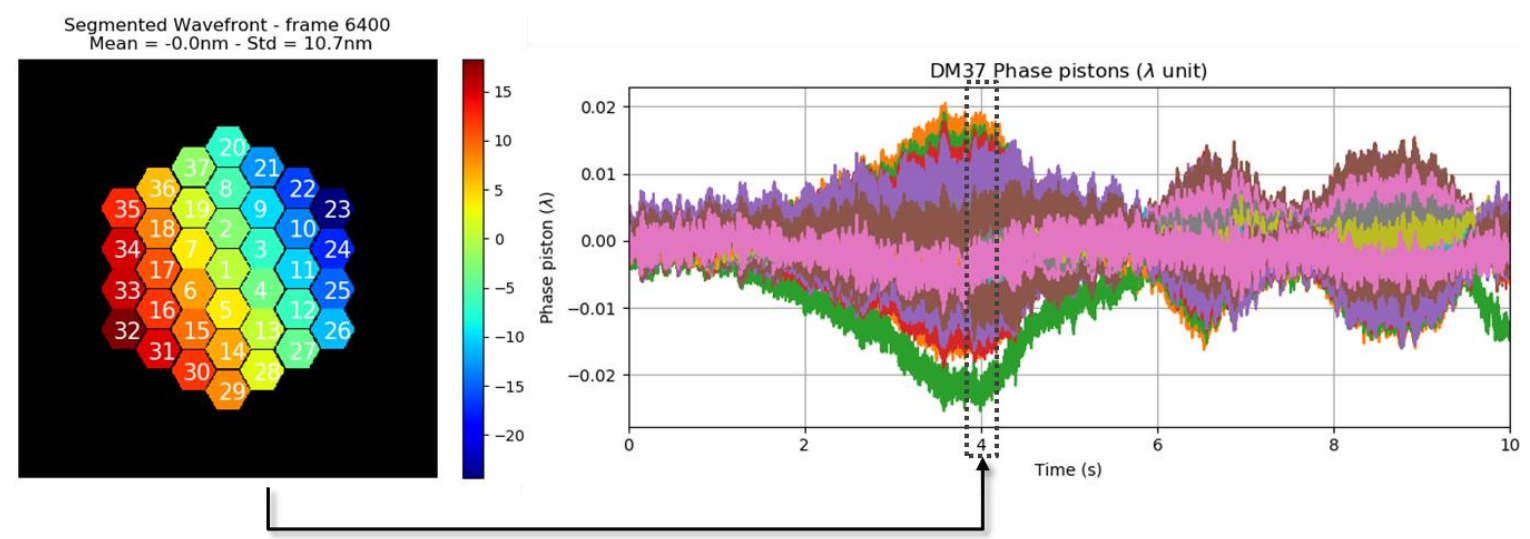

Figure 10. A piston per segment tracking diagnosis of the DM at rest (@ 1600 fps). Tip-tilt aberration (left panel) is the main erroneous wavefront sensed by the PISTIL interferometer. 

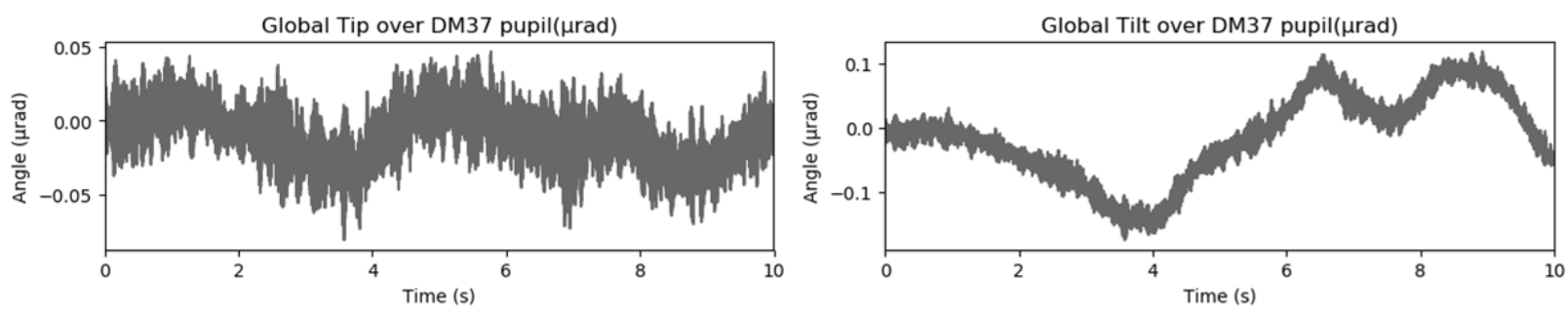

Figure 11.Global tip/tilt projection extracted from piston datasets.

Such tip/tilt aberration can also be tracked by the use of a piston correlation matrix, applied to all reconstructed piston values. The figure of merit displayed in Figure 12 clearly shows direct and opposite correlations of antipode pistons (example: segments 32-35 vs. segments 23-26).

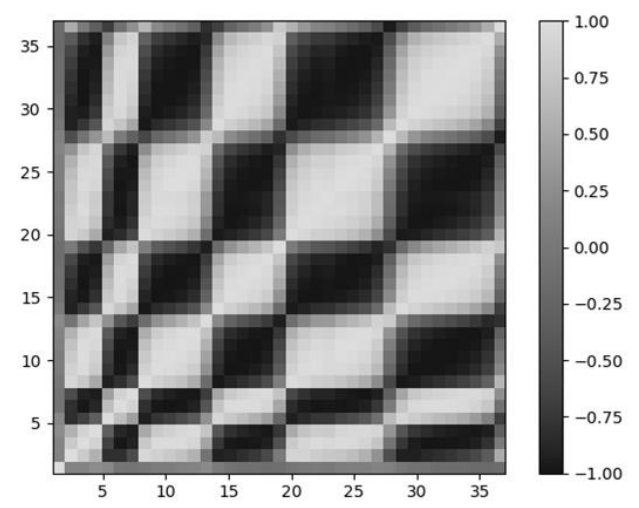

Figure 12. Figure of merit for tip/tilt detection using piston correlation matrix (x/y axis: piston number).

With the generalized matrix reconstruction used in the process, information redundancy allows to clearly separate piston sources and minimize cross-talk in noise propagation sense. Therefore, each piston power spectral density (PSD) is only the result of the channel's frequency content (Figure 13). As the mirror remains at electronically flat and at rest, it hints about noise source influences from the laboratory. Frequency clear distinction has also been confirmed by simulation.
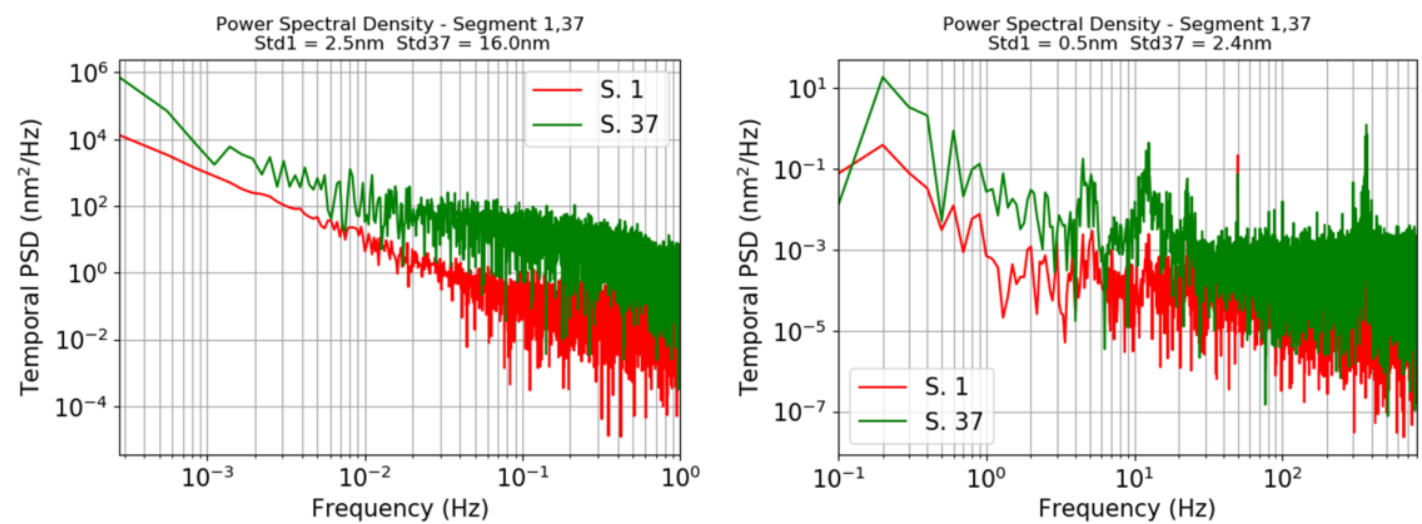

Figure 13. Power spectral densities for segment 1 and segment 37, in ]0-1Hz] and ]0,800Hz] regimes. Left panel: Segment 1 presents a clear $1 / f^{2}$ slope while segment 37 is more of a pink noise $(1 / f)$. Right panel: as noise result in visible tip-tilt, external pistons are sensed more excited than the central one, except for the common $50 \mathrm{~Hz}$ peak.

Finally, two-wavelength mode can also be of use for sensing static high-piston range wavefronts such as a segmented vortex, or any else wavefront shape that could be of use in CBC. In the example of Figure 14, vortex is excited from the mirror for wavelength $\lambda_{1}$ (red) from the bench. Using both $\lambda_{1}$ (red) and $\lambda_{2}$ (orange) pistilograms allows to unwrap the 
phases $[22,23]$ and acutely retrieve the DM shape (RMS of $\left.\lambda_{1} / 105\right)$. If this method appears more suitable to wavefront static metrology, it can also be used to pulsed regime CBC systems, as one can use a narrowband filter to pick out two separates wavelength from its spectrum.
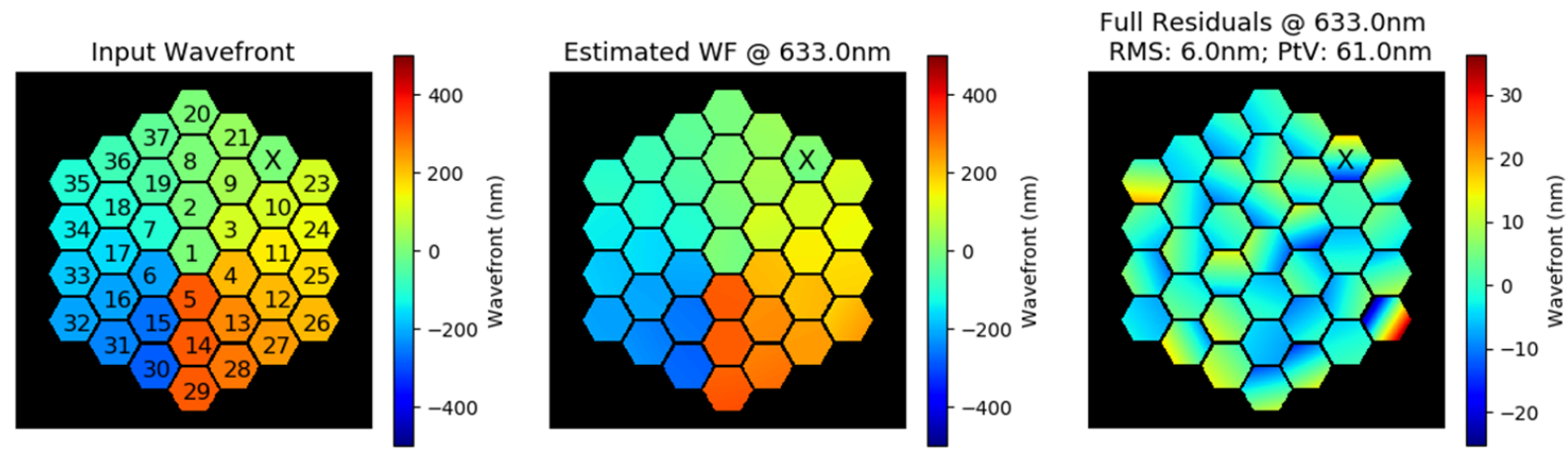

Figure 14. Segmented wavefront sensing for vortex wavefront. The $\mathrm{X}$ denotes an inactive segment. Peak-to-Valley (PtV) comes from erroneously tilted segment.

\section{CONCLUSION}

In this paper, we reviewed the principle of PISTIL interferometry and discussed its possible application to coherent beam combining (CBC) optical testbeds. In practice, the interferometer is modular: that to say, optics (lens) and optical elements (mask, grating, camera) can be interchanged rapidly and overall the system alignment is almost immediate: there is only one pistilogram position per optical configuration, related to the magnifications used. The interferometer does not need a reference arm for calibration. Depending on the situation, this calibration can be done by simulation referencing, laboratory referencing (with an optical flat, for example) or in situ auto-referencing (closed-loop reports).

We built a PISTIL demonstrator optical testbed, HIBISCUS, for science researches and industry-ready prototype manufactures. The bench is equipped with a segmented deformable mirror (DM) from IRIS-AO, extensively characterized. It allows preparing iterations of PISTIL interferometers for future research or industrial applications. An RMS wavefront value with natural tip-tilt wandering is of $\lambda / 200$ in average (dynamic analysis), and $\lambda / 880$ when this aberration is removed (static analysis). Those values validate the use of the PISTIL interferometer for segmented wavefront sensing in $\mathrm{CBC}$, whereas RMS requirements is often in between $\lambda / 20$ and $\lambda / 50$. Independent spectral analysis per sub pupil is enabled, as well as pattern wavefronts. Future work is direct application on CBC laser systems and proposal for new applications.

\section{FUNDING}

LabEx PALM (ANR-10-LABX-0039-PALM). 


\section{REFERENCES}

[1] Danson, C. N., et al., "Petawatt and exawatt class lasers worldwide," High Power Laser Sci. Eng. 7, e54 (2019).

[2] Mourou, G., Brocklesby, B., Tajima, T., et al. "The future is fibre accelerators," Nature Photon 7, 258-261 (2013)

[3] Fan, T. Y., "Laser beam combining for high-power, high-radiance sources," IEEE J. Quantum Electron. 11(3), 567-577 (2005).

[4] Arnaud, B. and Brignon, A., [Coherent Laser Beam Combining], Wiley-VCH Verlag GmbH \& Co. KGaA, (2013).

[5] Flores, A., Shay, T. et al., "Coherent beam combining of fiber amplifiers in a kW regime,” IEEE CLEO: 2011Laser Science to Photonic Applications, 1-2 (2011).

[6] Veinhard, M., Bellanger, S., et al., "Orbital angular momentum beams generation from 61 channels coherent beam combining femtosecond digital laser," Opt. Lett. 46, 25-28 (2021).

[7] Hou, T., Chang, Q., Chang, H., et al. "Structuring orbital angular momentum beams by coherent laser array systems with tip-tilt optimization," Results in Physics 19 (2020).

[8] Aksenov, V. P., Dudorov, V. V., Kolosov, V. V., Levitsky, M. E., "Synthesized Vortex Beams in the Turbulent Atmosphere", Frontiers in Physics 8 (2020).

[9] Adamov, E. V., Aksenov, V. P., Atuchin, V. V., Dudorov, V. V., Kolosov, V. V., Levitsky, M. E., "Laser beam shaping based on amplitude-phase control of a fiber laser array," OSA Continuum 4, 182-192 (2021).

[10] Liu, L., Vorontsov, M. A., "Phase-locking of tiled fiber array using SPGD feedback controller," Proc. SPIE 5895, 58950P (2005).

[11] Shay, T. M., Benham, B., "First experimental demonstration of phase locking of optical fiber arrays by RF phase modulation," Proc. SPIE 5550, (2004).

[12] Bellanger, C., Brignon, A., Colineau, J., Huignard, J. P., "Coherent fiber combining by digital holography," Opt. Lett. 33, 2937-2939 (2008).

[13] Antier, M., et al., "kHz Closed Loop Interferometric Technique for Coherent Fiber Beam Combining," IEEE JSTQE 20 (5) (2014).

[14] Prevost, F., Lombard, L., et al., "Coherent beam combining of narrow-linewidth long-pulse Er3+-doped multicore fiber amplifier,” Opt. Exp. 25 (9), 9528-9534 (2017).

[15] Kabeya, D., Kermène, V., et al. "Efficient phase-locking of 37 fiber amplifiers by phase mapping in an optimization loop," Opt. Exp. 25(12), 13816-13821 (2017).

[16] Bourderionnet, J. Bellanger, C., Primot, J., Brignon, A., "Collective coherent phase combining of 64 fibers," Opt. Exp. 19(18), 17053-17058 (2011).

[17] Fsaifes, I., et al. "Coherent beam combining of 61 femtosecond fiber amplifiers," Opt. Exp. 28(14), $20152-$ 20161 (2020).

[18] Chang, H. Chang, Q., et al., "First experimental demonstration of coherent beam combining of more than 100 beams," Photonics Research 8 (12), 1943-1948 (2020).

[19] Spencer, M., et al. "Piston phase compensation of tiled apertures in the presence of turbulence and thermal blooming," IEEE Aerospace Conference (2014).

[20]Deprez, M., Bellanger, C., et al., "Piston and tilt interferometry for segmented wavefront sensing," Opt. Lett. 41(6), 1078-1081 (2016).

[21] Deprez, M., Wattelier, B. et al., "Phase measurement of a segmented wave front using PISton and TILt interferometry (PISTIL),” Opt. Exp. 26 (5), 5212-5224 (2018).

[22] Rouzé, B., Bellanger, C., et al., "Segmented wavefront metrology using multicolor PISTIL interferometry", Proc. SPIE 11352, 113520V (2020).

[23] Rouzé, B., Primot, J., et al. "High-dynamic range segmented mirror metrology by two-wavelength PISTIL interferometry: demonstration and performance," Opt. Exp. 28 (22), 32415-32425 (2020).

[24] Takeda, M., Ina, H., Kobayashi, S., "Fourier-transform method of fringe-pattern analysis for computer-based topography and interferometry", J. Opt. Soc. Am. 72(1), 156-160 (1982).

[25] Penrose, R., and Todd, J. A., “A generalized inverse form matrices,” Math. Proc. Camb. Philos. Soc. 51(3), 406-413 (1955).

[26] Zamkotsian, F., Lanzoni, P., et al., "Operation of a MOEMS deformable mirror in cryo : Challenges and results," Micromachines 8(8), 233 (2017). 